\title{
Genetic covariance structure of growth in the salamander Ambystoma macrodactylum
}

\author{
GJ Ragland and PA Carter \\ School of Biological Sciences, Washington State University, Pullman, WA, USA
}

The size of an organism at any point during ontogeny often has fitness consequences through either direct selection on size or through selection on size-related morphological, performance, or life history traits. However, the evolutionary response to selection on size across ontogeny (a growth trajectory) may be limited by genetic correlations across ages. Here we characterize the phenotypic and genetic covariance structure of length and mass growth trajectories in a natural population of larval Ambystoma macrodactylum using function-valued quantitative genetic analyses and principal component decomposition. Most of the phenotypic and genetic variation in both growth trajectories appears to be confined to a single principal component describing a pattern of positive covariation among sizes across all ages. Higher order principal components with no significant associated genetic variation were identified for both trajectories, suggesting that evolution towards certain patterns of negative covariation between sizes across ages is constrained. The well-characterized positive relationship between size at metamorphosis and fitness in pond-breeding amphibians predicts that the across-age covariance structure will strongly limit evolution only if there is negative selection on size prior to metamorphosis. The pattern of genetic covariation observed in this study is similar to that observed in other vertebrate taxa, indicating that size may often be highly genetically and phenotypically integrated across ontogeny. Additionally, we find that phenotypic and genetic analyses of growth trajectories can yield qualitatively similar patterns of covariance structure.

Heredity (2004) 92, 569-578, advance online publication, 28 April 2004; doi:10.1038/sj.hdy.6800462

Keywords: quantitative genetics; growth trajectory; function-valued; Ambystoma; constraint; covariance function; evolution

\section{Introduction}

The way in which an organism grows can shape many aspects of complex phenotypes expressed over the course of ontogeny. Patterns of growth frequently determine age-specific resource utilization and ecological niche (Ebenman, 1992; Amara et al, 2001) and may mediate life history traits such as age and size at first reproduction. In some cases, growth may even alter the functional significance of morphological and performance traits during ontogeny (Gregory and Wood, 1998; Seibel et al, 1998; Koehl, 2000). Clearly, at the level of the individual, the phenotypic expression of a growth trajectory (ie, an ontogenetic trajectory of size) can have profound fitness effects (Travis, 1980; Ricklefs, 1984; Werner, 1986; Hutchings, 1993; Harfenist, 1995; Lankford et al, 2001); therefore, population-level variation in the form of a growth trajectory will often serve as a direct or indirect target of natural selection. As with any trait, the evolutionary response of a growth trajectory will be determined by not only the strength and mode of selection, but also by the amount genetic variation in a population, the genotype to phenotype mapping function, and genetic covariances with other traits (Falconer and Mackay, 1996). The added feature that makes growth trajectories

Correspondence: GJ Ragland, Department of Biology, University of North Carolina at Chapel Hill, CB \#3280, Coker Hall, Chapel Hill, NC 275993280, USA. E-mail: gragland@email.unc.edu

Received 20 February 2003; accepted 4 March 2004; published online 28 April 2004 interesting from a quantitative genetic perspective is the across-age covariance structure, or the relationship between size at any given age and size at all other ages. Characterizing this added dimension of covariation is an important step towards understanding how patterns of growth have been and can be shaped by natural selection.

Growth trajectories are one of a special class of quantitative traits, often termed 'infinite-dimensional' (Kirkpatrick and Heckman, 1989) or 'function-valued' (Pletcher and Geyer, 1999; Kingsolver et al, 2001), that cannot be described by a discrete measurement or set of measurements. Rather, they are an infinite series of traits defined over the range of an independent variable and are most accurately described as a function of that continuous independent variable. In general, the independent variable of a function-valued trait may be temporal, as in a growth trajectory, environmental, as in a reaction norm, or shape, as for morphology (Kirkpatrick et al, 1990). Following this convention, a growth trajectory is described as some measure of size as a function of age. If a growth trajectory is visualized as a $2 \mathrm{D}$ plot of size versus age, variation in a population of growth trajectories can be visualized as differences in the shape (ie, slope or curvature) or position (ie, intercept) of the trajectory. Treating sizes across ages explicitly as a function, variation for growth trajectories can be described by a covariance function (variance/covariance for size as a function of age), the continuous analog of a covariance matrix (variance/covariance between sizes at landmark ages).

An interesting prediction that arises from the infinitedimensional model is that function-valued traits may 
often exhibit a high degree of genetic constraint (Kirkpatrick and Lofsvold, 1992). The magnitude of the average genetic correlation necessary to produce an absolute constraint (a constraint that precludes evolution in a certain direction) for any set of correlated characters is approximately inversely proportional to the number of characters that covary. Given the continuous form (and thus infinite number of potential genetic correlations) of a function-valued trait, even small pairwise genetic correlations across values of the independent variable (eg, correlations between sizes across ages for a growth trajectory) may produce strong genetic constraints (Kirkpatrick and Lofsvold, 1992). This suggests that across-age covariance structure may play an important role in determining how growth trajectories evolve.

There is a fairly extensive body of literature on the quantitative genetics of growth in laboratory and domesticated animals (Roberts, 1961; Timon and Eisen, 1969; Atchley and Rutledge, 1980; Cheverud et al, 1983; Leamy and Cheverud, 1984; Kirkpatrick and Lofsvold, 1992; Meyer, 1998a; Rocchetta et al, 2000), but few studies have attempted to quantify the genetic component of phenotypic variation for growth in natural populations (but see Björklund, 1997; Badyaev and Martin, 2000). Here we explore quantitative genetic variation for growth trajectories in a natural population of the longtoed salamander (Ambystoma macrodactylum columbianum) in order to (1) characterize the genetic covariance structure of growth in a natural population and (2) identify any constraints that genetic architecture may place on the evolution of the growth trajectories.

Amphibians such as $A$. m. columbianum that metamorphose from aquatic larval stages to terrestrial adult stages provide an excellent system for empirically investigating the quantitative genetics of growth trajectories that have obvious fitness consequences. Larval growth terminates in metamorphosis, and larval growth trajectories will influence size at metamorphosis, and in some cases time to metamorphosis (Wilbur and Collins, 1973; Collins, 1979; Alford and Harris, 1988). Both timing and size at metamorphosis are important life history traits in pondbreeding amphibians that can have substantial fitness consequences. Size at metamorphosis can influence juvenile survival, size at first reproduction, and age at first reproduction (Semlitsch et al, 1988; Beck and Congdon, 1999), all of which are important fitness components.

In this study, we apply function-valued analyses to growth trajectories of mass and length of the larval stage of A.m. columbianum. Genetic and phenotypic covariance functions are estimated for each growth trajectory, and principal components associated with the genetic covariance functions are generated in order to identify major patterns of covariation. As the study population breeds in an ephemeral pond, metamorphic timing is an important life history trait that may be correlated with the endpoint of the growth trajectory (size at metamorphosis); hence genetic correlations between size and age at metamorphosis are also estimated.

\section{Methods}

\section{Study organism}

A. m. columbianum is a broadly distributed species of Ambystomatid salamander found in various habitats from southeastern Alaska to eastern Washington and Oregon and central Idaho (Petranka, 1998). Adult and juvenile stages are primarily terrestrial, but breeding activity and larval stages are limited to nonriverine aquatic environments. In eastern Washington populations of $A$. m. columbianum are typically distributed near permanent or ephemeral ponds in which breeding occurs seasonally. Mature adults begin migrating into ponds in mid-winter (late January to early February), and most breeding is complete by mid-March (G. Ragland, personal observation). Fertilization is internal, and sperm are transferred by means of a spermatophore deposited on the substrate by a courting male. After fertilization, females deposit eggs on vegetation and other available substrates. Development time through metamorphosis can be highly variable (50 days to 3 years) depending on environmental factors such as pond duration (Leonard et al, 1993).

\section{Field sampling and laboratory breeding}

The population sampled for this study is located on Smoot Hill Biological Preserve in Whitman County near Pullman, Washington. Breeding at this locality occurs in a small, temporary pond approximately $20 \mathrm{~m}$ in diameter at maximum water level. Drying times for the pond are highly variable from year to year, ranging from early June to late August (G Ragland, personal observation; JL Payne, personal communication, 2000).

From February to March of 2000, mature male and female $A$. $m$. columbianum were captured with drift fence/pitfall trap arrays as they migrated toward the pond for breeding. All captured individuals were brought to the laboratory and housed individually in clear plastic 'shoebox' containers placed in an environmental chamber maintained at $13.5^{\circ} \mathrm{C}$ with a constant 12:12 $\mathrm{h}$ photoperiod. Within a day of capture, containers housing females were filled with dechlorinated water, leaf-litter, and gravel substrate. Females were allowed to habituate in the aquatic container for 1-week in order to ensure that fertilization had not already occurred. Female $A$. $m$. columbianum are known to store sperm (Sever and Brizzi, 1998), therefore sperm from previous mating events can potentially fertilize eggs. A female was never observed to lay eggs during this time period, so it was assumed that all females in this study were not using stored sperm for fertilization. After the weeklong habituation period, a single male was introduced to each female enclosure for 1 week. The female was allowed an additional week to deposit eggs before all eggs were transferred to a separate container.

\section{Animal maintenance and measurement}

After being deposited, each clutch was relocated to a separate clear plastic container $(230 \times 110 \times 115 \mathrm{~mm})$ and the water was changed in each container biweekly until the eggs hatched. Variation in hatching success and early life mortality between clutches produced variation in clutch size across families (mean number of individuals per $\operatorname{clutch}=10$, maximum number per $\operatorname{clutch}=19$, minimum number per clutch $=4$, total number of individuals $=126$ ).

Larvae were housed individually within 2 weeks of hatching, at which point feeding commenced. For the duration of the experiment, larvae were fed 
Artemia (brine shrimp) ad libitum every third day. At 2 days after feeding, the water in each container was changed; excess Artemia were always observed in the discarded water. The environmental chamber in which the larvae were housed was maintained at $13.5^{\circ} \mathrm{C}$ and $12: 12 \mathrm{~h}$ photoperiod for the duration of the experiment. Pond temperatures in the field can become significantly higher than this during the summer months when the pond level is low, but changing the temperature of the environmental chamber during the experiment would have resulted in differential environmental effects among clutches caused by the variation in the date of egg deposition between clutches. An average temperature of $13.5^{\circ} \mathrm{C}$ is realistic for the sampled pond in years where the pond persists through August.

For a period of time after hatching, larval $A . m$. columbianum are too small and fragile to be handled without damage. Measurement of snout-vent length (SVL) commenced 3 months after the last clutch had completely hatched. Measurements of body mass incurred more damage to smaller animals, so mass measurements began 2 weeks later than the first week of SVL measurements. Mass and SVL were measured for each individual once per week (mass to the nearest milligram, SVL to the nearest tenth of a millimeter), from the time of the initial measurement through the completion of metamorphosis. Mass, SVL, and age (number of days from egg deposition to metamorphosis) were recorded at the completion of metamorphosis. Metamorphosis was considered complete when the gills were completely resorbed. A small number of individuals $(n=13)$ died very close to metamorphosis, but since the time to completion of metamorphosis was very consistent and predictable based on observation of the degree of resorption of the gills, age at metamorphosis was extrapolated in these instances. Growth trajectories and age at metamorphosis for individuals with extrapolated ages did not differ significantly from individuals that completed metamorphosis. Mass and SVL at metamorphosis for individuals with extrapolated ages were recorded as missing values.

\section{Quantitative genetic model}

Methods for quantifying the parameters of a discrete quantitative trait or set of traits have been well described (Lande, 1979; Falconer and Mackay, 1996; Lynch and Walsh, 1998), and these methods can be extended to function-valued traits (eg, Cheverud et al, 1983; Via and Lande, 1985). A major limitation of these multivariate methods, however, is that genetic parameters of a function-valued trait can only be estimated for the discrete points of the independent variable at which measurements were taken. Here we implement analyses that model variances and covariances as a covariance function. This model allows interpolation between values of the independent variable at which measurements were taken, accounts for the distance between measurements, and incorporates the ordinal nature of the independent variable. For the current analyses covariance between size (mass or SVL) at age $A$ and size at age $\theta$ are modeled as the function $C(A, \theta)$. We estimate this function for phenotypic covariance (the $P$-function) and genetic covariance (the G-function). Specifically, we use the orthogonal polynomial model described by Kirkpatrick et al (1990) and Meyer (1998a).

As with covariance matrices, the patterns of variation in a covariance function may be summarized by principal components (PCs) that represent uncorrelated major axes (or directions) of variation in the data. Similar to the PCs of a covariance matrix, the amount of variation associated with each PC of a covariance function is indicated by the associated eigenvalues, which are typically ordered from largest to smallest. The patterns of loadings on each PC of a covariance function are described by the eigenfunctions (ordered according to the eigenvalues with which they are associated), the continuous analogs of eigenvectors. In the current analyses, the loading values of an eigenfunction indicate both the variance within that axis accounted for by size at each age (the magnitude of the loading value) and the direction of covariation between sizes across ages within that axis (the sign of the loading value). Generally, eigenfunctions are most useful for visualizing major patterns of covariance between sizes across all ages in the data set. If the loading values for two ages each display the same sign this indicates positive covariation between sizes at those ages, while opposite signs indicate negative covariation.

\section{Quantitative genetic analyses}

Ages were standardized for all analyses as percentage of elapsed time to metamorphosis (calculated as [age at measurement/age at metamorphosis]) because of the variation in the date of egg deposition among families. In order to simplify the models, ages were grouped into ranges of $5 \%$ of the larval period (\%LP), and multiple measurements within each range for each animal were averaged. This simplification yielded size averages centered at seven ages: 67.5\%LP, 72.5\%LP, 77.5\%LP, 82.5\%LP, 87.5\%LP, 92.5\%LP, and 97.5\%LP. Data for mass measurements were restricted to the final six ages as a result of the discrepancy in the time of initial measurement between mass and SVL. There was considerable variation in the number of individuals measured per age (Table 1) caused by variation in development rate, variation in the date of oviposition, and some late-life mortality, although the relative representation of families across ages was fairly uniform. We used the maximum likelihood estimation methods described below largely because likelihood-based estimates are much more robust than least-squares estimates for unbalanced data (Shaw, 1987).

We attempted to create a composite index of overall size by performing a conventional principal components analysis on mass and SVL, but this analysis failed to produce a PC that explained a large percentage of the variation in both mass and SVL. Consequently, we treated each trait separately in the quantitative genetic analyses.

Procedures for estimating genetic covariance functions assume a multivariate normal distribution for the set of traits to be analyzed (Meyer and Hill, 1997). All data were checked for normality using a Shapiro-Wilk test. Normality of the entire data set was tested for SVL at metamorphosis, mass at metamorphosis, age at metamorphosis, and SVL and mass at each of the ages specified above. All data were found to be normal 
$(P>0.10)$ with the exception of deviations from normality within 3 of the ages for mass. Estimations of genetic variances for mass for each of the age ranges were obtained using an ANOVA model in which residuals were normally distributed. Genetic variances calculated from the estimated covariance function for mass were very consistent with values estimated in the ANOVA model, indicating that departures from normality did not seriously affect the estimate of the covariance function.

Genetic correlations (and approximate standard errors) between mass and SVL at metamorphosis and age at metamorphosis were estimated using a restricted maximum likelihood (REML) procedure in conjunction with a multivariate model (Meyer, 1991), while the genetic covariance functions, principal components, and eigenvalues of the growth trajectories for mass and SVL were estimated using the REML procedure in conjunction with a function-valued model (Meyer, 1998a). All analyses were implemented in the program DfReml (Meyer, 1998b). The orthogonal polynomial model for the genetic covariance function and the REML estimation procedure have been well described elsewhere (Kirkpatrick and Heckman, 1989; Kirkpatrick et al, 1990; Meyer, 1998a), so we provide only a brief explanation here. Note that although we used the orthogonal polynomial model described by Kirkpatrick et al. (1990), we used a procedure that estimates the covariance function directly from the data and does not require the intermediate estimation of the covariance matrix (Meyer, 1998a).

The parameters of the genetic covariance function were estimated by implementing an REML algorithm to maximize the likelihood function of a random regression model that included genetic and permanent environmental effects. Covariance functions for both genetic and permanent environmental effects were modeled on an orthogonal polynomial basis set and the 'average information' REML algorithm (Meyer, 1998a) was used to maximize the likelihood function. Only the estimated genetic covariance functions are presented here. To estimate phenotypic covariance functions we used the same procedure described above except that only a single animal effect was modeled (ie, family effects were disregarded) (Meyer, 1998b).

The Bayesian information criterion (BIC) was used in a backward selection process to select the most parsimonious model for each covariance function. This selection criterion produces a BIC score based on the calculation:

$$
\mathrm{BIC}=-2(\text { maximized } \log \text { likelihood })+p \ln (n)
$$

where $p$ is equal to the number of parameters in the model and $n$ is the sample size (Agresti, 1990). Full fit models (where the order of polynomial fit is equal to the number of ages in the data) were compared to reduced order of fit models in this manner in order to select the most parsimonious model that captured the majority of the variation in the data.

PCs (eigenvalues and associated eigenfunctions) of the covariance functions were calculated as in Kirkpatrick et al (1990). The number of PCs estimated for each covariance function was equal to the order of polynomial fit (Kirkpatrick et al, 1990). Uncertainty in the estimates of the eigenvalues was assessed by bootstrap analysis. Eigenvalues were estimated (in DFREML) for 1000 bootstrap replicate data sets (resampling with replacement across families) and bootstrap bias correction was used to adjust for estimation bias (Shao, 1988). The $95 \%$ confidence intervals were calculated for these biasadjusted estimates. In order to test whether eigenvalues smaller than the leading eigenvalues were significantly different from zero in a likelihood framework, the likelihood was maximized for models in which eigenvalues were systematically set to zero, from the smallest to the largest. Likelihood ratio test statistics were then constructed to hierarchically test the significance of each eigenvalue.

All genetic covariance functions and genetic correlations were estimated from a full-sib breeding design, and therefore may contain both additive and nonadditive genetic variance. The inclusion of nonadditive effects into the estimates of genetic variation is potentially problematic because additive and nonadditive effects are confounded. Fortunately, previous studies provide estimates of nonadditive genetic variances for amphibian life history traits related to larval growth (Travis et al, 1987; Newman, 1988); the results of these studies indicate that there may be a certain degree of upward bias in genetic parameters for growth and related life history traits estimated in an analysis of full sibs, but the contribution of non-additive compared to additive effects is relatively small.

\section{Results}

\section{Growth trajectories}

Table 1 presents the mean, standard deviation, and sample size for each age, and Figure $1 \mathrm{a}$ and b shows the estimated overall mean and family mean growth trajectories for mass and SVL, respectively. Both overall mean trajectories display a trend of roughly linear increase in size with increasing age up to $92.5 \% \mathrm{LP}$, but after this point mass decreases for the duration of larval growth. This decrease in mass is typical for the final stages of larval growth in salamanders and results from the abrupt morphological changes accompanying metamorphosis. The family mean growth trajectories are representative of the genetic variation about the mean trajectory, but this variation is an approximation of the actual genetic variation because among family variances were weighted by family size in the analyses. When describing the results of the function-valued analyses in terms of variation in growth trajectories, we frequently refer to genetic variation as variation for family mean trajectories and phenotypic variation as variation for individual growth trajectories.

\section{Covariance functions}

Surface plots of the estimated phenotypic and genetic covariance functions (both fifth-order polynomial fit) for mass are shown in Figure $2 a$ and $b$. The interpretation of covariance functions in general is similar to that for covariance matrices. The diagonal trend of the covariance function is analogous to the variances along the diagonal of a covariance matrix. Thus, the diagonal trends for both the mass $P$-function and $G$-function indicate that variance (both phenotypic and genetic) generally increased with increasing age. Again drawing the parallel between covariance matrices and functions, 
Table 1 Number of individuals measured $(n)$, mean $(\bar{x})$, and standard deviation $(\hat{\sigma})$ for mass and SVL at each of the measured ages (\% LP = percent of larval period).

\begin{tabular}{|c|c|c|c|c|c|c|}
\hline Age (\%LP) & 67.5 & 72.5 & 77.5 & 82.5 & 87.5 & 92.5 \\
\hline$n$ & $29^{*}$ & 58 & 101 & 119 & 124 & 125 \\
\hline $\bar{x}, \operatorname{SVL}\left(\mathrm{m}^{-4)}\right.$ & 146 & 167 & 178 & 192 & 202 & 210 \\
\hline$\hat{\sigma}, \mathrm{SVL}$ & 16.8 & 13.8 & 13.4 & 10.6 & 10.0 & 11.1 \\
\hline $\bar{x}$, Mass $\left(\mathrm{g}^{-2}\right)$ & NA & 26.0 & 31.6 & 36.3 & 41.3 & 45.8 \\
\hline$\hat{\sigma}$, Mass & NA & 4.72 & 5.02 & 4.42 & 4.76 & 6.08 \\
\hline
\end{tabular}

Only SVL was measured at $67.5 \%$ LP (see Methods).

a

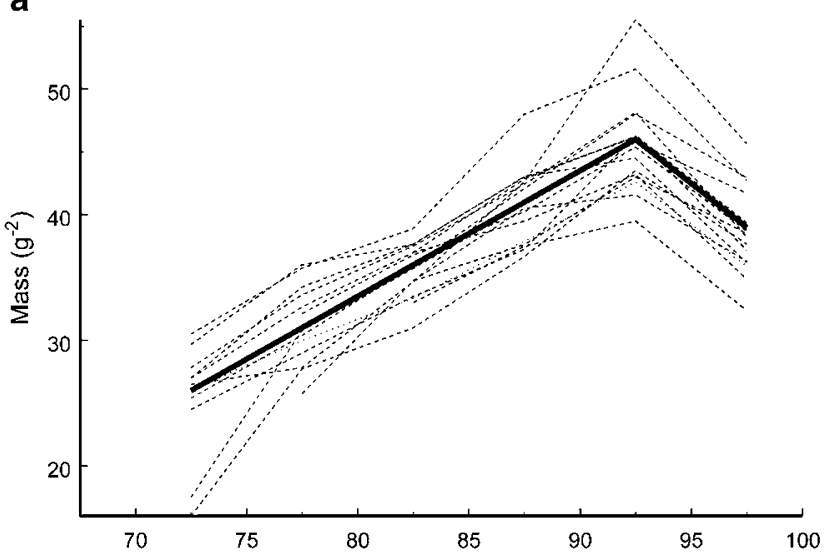

b

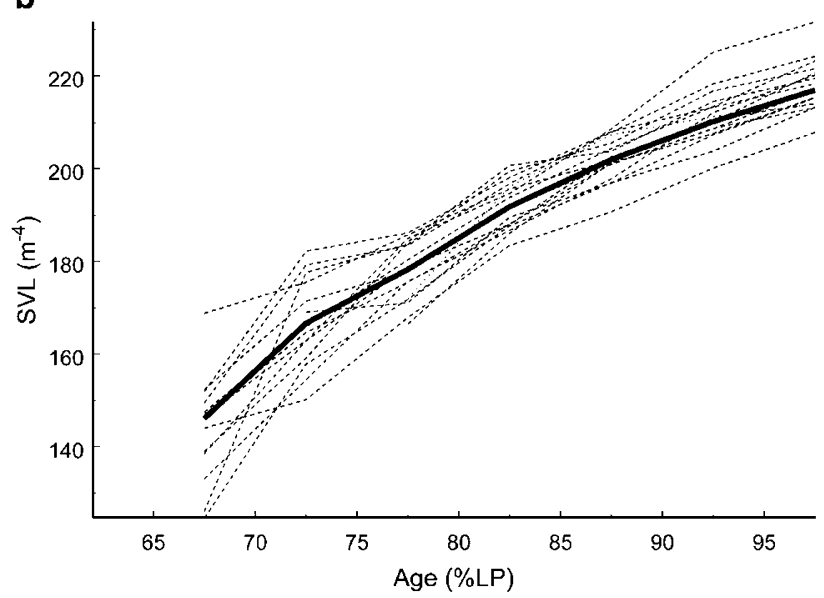

Figure 1 Estimated population mean (solid line) and family mean (dashed lines) growth trajectories for mass (a) and SVL (b).

points on the surface off the diagonal represent covariance between mass at one age and mass at another age. Genetic covariances were mostly positive (there were slightly negative covariances between the pairs of ages 77.5-92.5\%LP, 77.5-97.5\%LP, and 82.5-97.5\%LP) and were at their highest (most positive) values between ages later in the larval period. Phenotypic covariances were all positive and also attained their highest values at later ages. The curvature about the diagonal indicates that both phenotypic and genetic covariance decreased with increasing separation between ages. This result is fairly intuitive because the mass of an actively growing individual is expected to be less predictable over long time scales than over short time scales. Overall, the patterns of covariance in the $P$ - and $G$-function are nearly identical, although the G-function does display a bit more curvature and reaches its maximum at $92.5 \% \mathrm{LP}$, while the $P$-function reaches its maximum at $97.5 \% \mathrm{LP}$.

The phenotypic and genetic covariance functions (both fourth order polynomial fit) for SVL are shown in Figure $2 \mathrm{c}$ and $\mathrm{d}$. In contrast to the results for mass, phenotypic and genetic variance for SVL (the diagonal trend) were greatest at early ages, decreased through intermediate ages, and increased slightly for the final 5\%LP. Similar to the results for mass, phenotypic and genetic covariances for SVL decreased with increasing distance between ages. As with mass, the $P$ - and G-functions for SVL yielded similar patterns, but phenotypic variance (and covariance) declined rapidly at ages later than $67.5 \% \mathrm{LP}$, while genetic variance remained relatively high until reaching a minimum at approximately 92.5\%LP. Taken together, the patterns of phenotypic covariance for mass and SVL suggest that relative to the population mean, individuals that are large at one age also tend to be large at all ages, and individuals that are small at one age tend to be small at all ages. The patterns of genetic covariance for mass and SVL suggest the same trend for family means.

\section{Principal components}

The ordered eigenvalues (bias adjusted) and their corresponding 95\% confidence intervals for the mass and SVL P- and G-functions are shown in Figure 3. The relative magnitudes of the eigenvalues indicate that the majority of both genetic and phenotypic variance in both the mass and SVL growth trajectories is accounted for by the PCs associated with the first eigenvalue, and to a lesser extent the second eigenvalue. Relative to the magnitude of the first two eigenvalues, most of the higher order eigenvalues were estimated as effectively zero. Likelihood ratio tests indicated that none of the eigenvalues beyond the leading eigenvalue from the genetic analyses of both mass and SVL were significantly different from zero. For the phenotypic analyses, likelihood ratio tests indicated that only the first three eigenvalues (again for both mass and SVL) were significantly different from zero. Qualitatively the results of the phenotypic and genetic analyses are comparable, and the differences in the significance of the eigenvalues may have been caused by increased sampling error in the genetic analyses (ie fewer degrees of freedom). The two major patterns that emerge from these results are (a) that principal component decomposition yields strikingly similar results for phenotypic and genetic analyses, and (b) that most of the variation in mass and SVL growth trajectories is confined to only a few dimensions. 

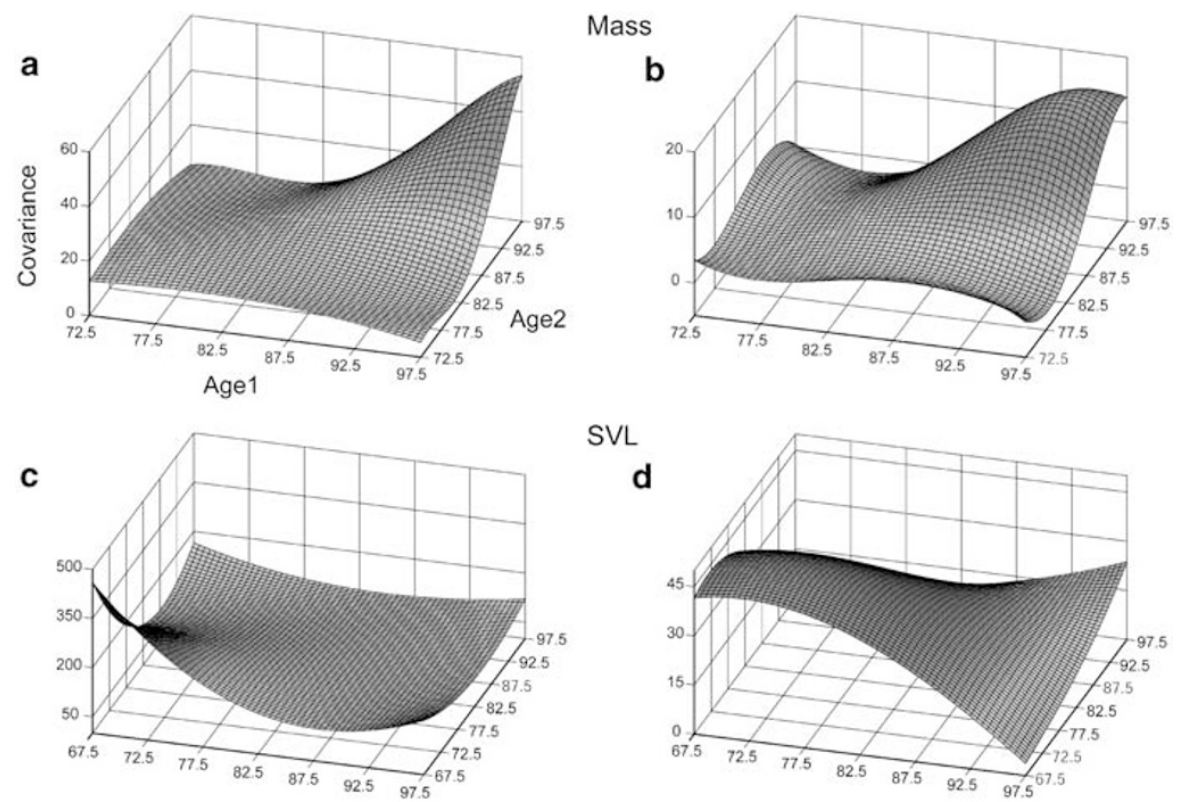

Figure 2 Phenotypic and genetic covariance functions of the mass $(a, b)$ and SVL $(c, d)$ growth trajectories. Each covariance function takes the general form $C(A, \theta)$, where 'Age1' and 'Age2' correspond to age $A$ and age $\theta$. $C(A, \theta)$ is equal to either phenotypic covariance (a, c) or genetic covariance $(\mathrm{b}, \mathrm{d})$. Original measurement units were $\mathrm{g}^{-2}$ (mass) and $\mathrm{m}^{-4}(\mathrm{SVL})$.

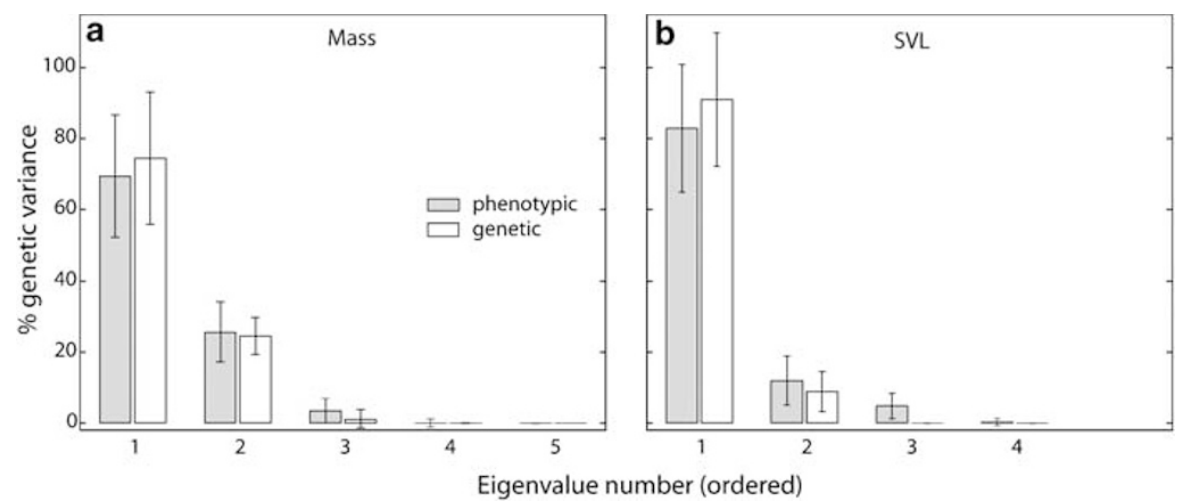

Figure 3 Ordered (largest to smallest) eigenvalues of the phenotypic covariance function (shaded bars) and the genetic covariance function (white bars) for both mass (a) and SVL (b). Error bars represent 95\% confidence intervals based on bootstrap resampling.

The PCs associated with the largest eigenvalues summarize the major patterns of variation in the data (while PCs associated with zero eigenvalues describe patterns of variation that are not present in the data). Since we are interested primarily in variation that can respond to selection, we present only the PCs from the genetic analyses here, although PCs from the phenotypic analyses were qualitatively similar in all cases. The first and second PCs for mass and SVL are presented because together they describe $99 \%$ of the variation for both growth trajectories. Recall that when we refer to PCs here, we are referring to the continuous eigenfunctions produced from the principal component decomposition of a continuous covariance function.

The first PC of the mass G-function accounted for $74.5 \%$ of the genetic variation in the growth trajectory. The loading values are all positive (Figure 4a), indicating positive genetic covariation across all ages within this axis of variation (loading values at all ages have the same sign). The trend towards increasing loadings with increasing age closely tracks the diagonal trend (increasing genetic variance with increasing age) of the corresponding $G$-function (Figure $2 b$ ). The variation described by this PC corresponds to variation for family mean trajectories that are either above or below the population mean trajectory at all ages (ie, variation in the intercept of the trajectory). The second PC of the mass G-function accounted for $24.6 \%$ of the genetic variation in the growth trajectory and displays loading values that are highly negative for ages early in the larval period and highly positive for ages near metamorphosis (Figure 4a). This pattern indicates negative genetic covariation between mass at early ages and mass at ages late in the larval period (loading values at early ages and late ages have different signs). The variation described by this axis is for family mean growth trajectories that are above the population mean trajectory at early ages and below the population mean trajectory at late ages, or vice versa 


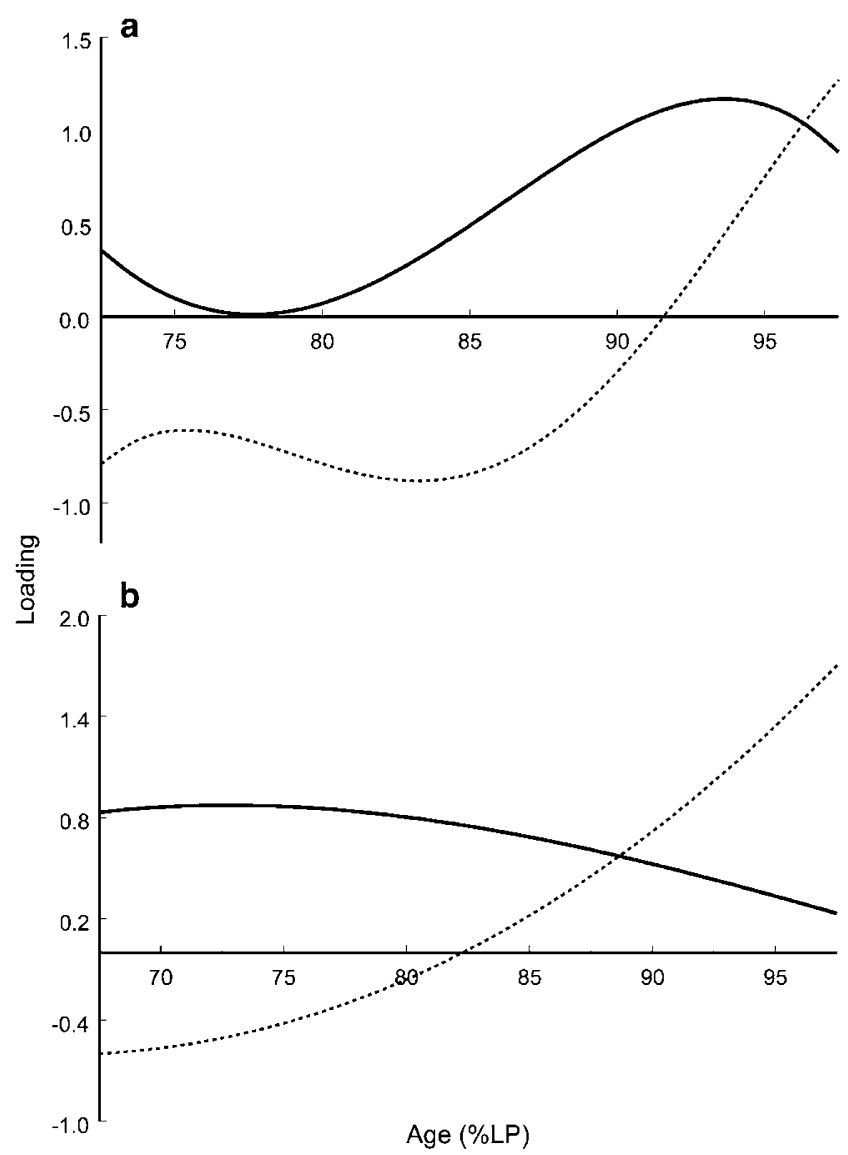

Figure 4 First (solid line) and second (dashed line) principal components of the mass (a) and SVL (b) genetic covariance functions. Each point along the PCs indicates the loading value for mass or SVL at a particular age.

(ie, variation for family mean growth trajectories that cross the population mean growth trajectory).

Similar to mass, the first PC of the SVL G-function accounted for a large proportion of the genetic variation $(91.0 \%)$ and displays positive loading values for all ages, indicating positive covariation between SVL at all ages within this axis of variation (Figure $4 \mathrm{~b}$ ). As with mass, the first PC for SVL closely resembles the diagonal trend in the corresponding G-function (Figure 2d). This PC describes variation for the intercept of family mean trajectories similar to that described for the first PC for mass, but there is proportionately more variation in SVL at early ages rather than at late ages. The second PC for SVL closely resembles the second PC for mass, with negative loading values at early ages and positive loading values at ages near metamorphosis (Figure 4b). The second PC accounted for $8.90 \%$ of the genetic variation in SVL and describes negative genetic covariation between SVL at early ages and late ages in the larval period. The variation about the mean growth trajectory corresponding to this axis is similar to that previously described for the second PC for mass.

The patterns of variation in family mean growth trajectories depicted in Figure $1 \mathrm{a}$ and $\mathrm{b}$ serve as a visual confirmation of the results from the principal components analysis. Indeed, most of the family mean trajectories of both mass and SVL are either above or below the population mean growth trajectory at all ages (variation described by the first PC), while relatively few family mean trajectories cross the population mean trajectory (variation described by the second PC).

\section{Genetic correlations}

Age at metamorphosis was positively genetically correlated with SVL at metamorphosis $\left(r_{\mathrm{g}}=0.471 \pm 0.0319\right)$ and highly positively genetically correlated with mass at metamorphosis $\left(r_{\mathrm{g}}=0.749 \pm 0.165\right)$, suggesting that body size at metamorphosis was positively influenced by the length of the larval period.

\section{Discussion}

The estimated growth trajectories for both mass and SVL in the sampled population of $A$. m. columbianum display genetic variation available for evolutionary change, although the patterns of variations differ between the two. Both phenotypic and genetic variance in mass increased with increasing age, while phenotypic and genetic variance in SVL decreased with increasing age. The cause of this difference is not entirely clear, although it is possible that there is a threshold size for metamorphosis associated predominantly with length rather than mass, such that all individuals raised under similar environmental conditions reach a similar target length at metamorphosis. Experimental evidence for threshold sizes at metamorphosis in larval amphibians exists (Alford and Harris, 1988; Tejedo and Reques, 1994; Beck, 1997), but generally mass and length are used interchangeably as a measure of overall size so their relative effects cannot be disentangled. Experimental manipulation of growth rates would be required to test explicitly whether there is a threshold size for metamorphosis in A. m. columbianum, but our results suggest that length and mass may interact differently with metamorphic timing.

Regardless of the differences in the form of the covariance functions, both growth trajectories have the potential for evolutionary responses, although the results indicate that genetic covariance structure may constrain the direction of evolutionary response. The first PC for both mass and SVL, describing a pattern of positive covariation among sizes across all ages, accounted for the majority of the genetic variation in both growth trajectories. This result can be confirmed by evaluating the mass and SVL G-functions at the measured ages and using the extracted variances and covariances to calculate all pairwise genetic correlations between the measured ages (equivalently, the genetic correlation functions could be calculated from the covariance functions and evaluated at the measured ages). The average genetic correlation between the measured ages is 0.46 for mass and 0.74 for SVL, confirming that, on average, there is positive genetic covariation for size across all ages. Collectively, this evidence suggests that the population mean growth trajectory for both mass and SVL is relatively unconstrained to evolve towards either increased or decreased mass and SVL at all ages in the larval stage. In fact, the maximum response to selection of the growth trajectories will be elicited by a selection gradient function (the continuous analog of a selection gradient vector) proportional to the first PCs (Kirkpatrick and Lofsvold, 1992). Evolution in directions described by 
PCs with decreasing associated eigenvalues, however, will be increasingly limited.

Theoretically, PCs associated with zero eigenvalues represent absolutely constrained directions of evolution, although as Kirkpatrick and Lofsvold (1992) argue, it is not possible to identify definitively absolute constraints in an empirical study. In keeping with this argument and acknowledging our limited sample size, we do not attempt to equate PCs associated with nonsignificant eigenvalues with absolute constraints. Our results do suggest, however, that there is relatively little genetic variation for the shape of both growth trajectories compared to genetic variation for the overall elevation (ie, position) of the trajectories (described by the first PC), although a modest amount of genetic variation is associated with patterns of negative covariation between size at early and late ages (described by the second PC). Based on the large disparity between the amounts of genetic variation accounted for by the first two PCs $(\sim 99 \%)$ and the third and higher order PCs $(<0.9 \%)$ of both growth trajectories we can predict that evolutionary responses will be strongly deflected towards the patterns of covariation described by the first PC, and to a lesser extent the second PC (Björklund, 1997; Arnold et al, 2001; Steppan et al, 2002).

As the first PC for both mass and SVL describes a pattern of positive covariation among sizes across all ages, if selection in the natural population of larval $A$. $m$. columbianum favors increased or decreased size at all ages then evolutionary responses should be relatively unconstrained. Strong empirical evidence for the positive relationship between size at metamorphosis and fitness for larval amphibians (Semlitsch et al, 1988; Beck and Congdon, 1999) suggests that selection will act to increase size at the termination of the larval growth trajectory, and if there is positive selection for size at all other ages, then evolutionary responses should be strong and in the direction of selection. If, however, selection is positive at some ages and negative at others, then evolutionary responses should be relatively weak and in a direction oblique to the direction of selection. Evolutionary responses will be particularly constrained in the case where selection gradient functions are approximately proportional to linear combinations of the third and higher order PCs. For example, a negligible amount of genetic variation was associated with the third PC for SVL (Figure 5a): thus, there is little to no genetic variation for growth trajectories that have values for SVL larger than the population mean at early and late ages and values lower than the population mean at intermediate ages, or vice versa (Figure $5 b$ ). Figure $5 c$ depicts patterns of selection for increased SVL at early and late ages and decreased SVL at intermediate ages, or vice versa. Since effectively there is no genetic variation of this sort to select upon (ie, selection is proportional to the third PC), no evolutionary response would be expected.

In addition, constraints on evolutionary responses may be imposed by the genetic correlations between the terminus of the larval growth trajectory (size at metamorphosis) and age at metamorphosis. As the study population inhabits a temporary pond, age at metamorphosis is almost certainly under strong negative directional selection during dry years because of mortality caused by desiccation (eg, Carter, 1997). Therefore, the strong positive genetic correlations between both mass
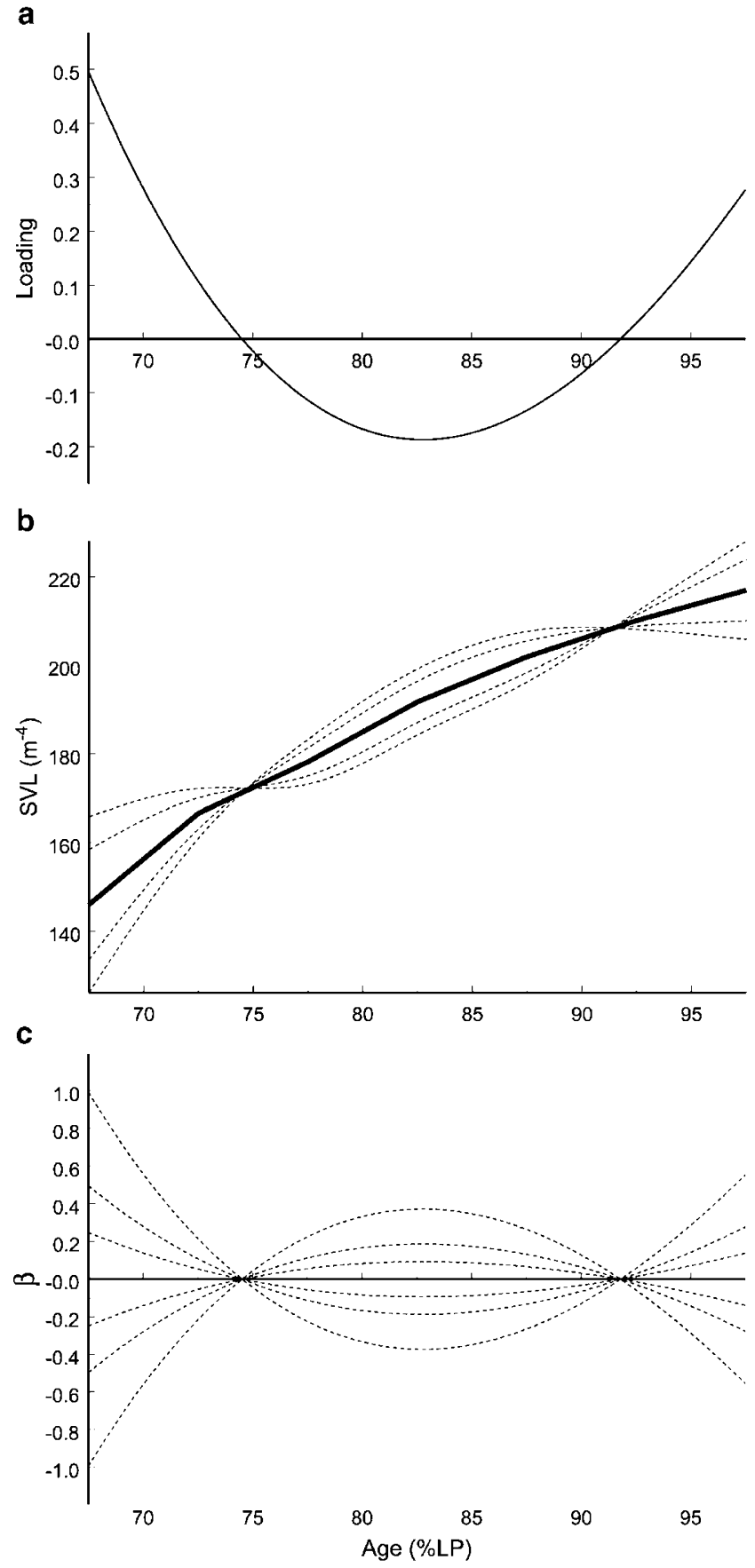

Figure 5 Third principal component of the SVL genetic covariance function (a), hypothetical family mean growth trajectories (dashed lines) whose variation about the estimated population mean growth trajectory (solid line) is described by this principal component (b), and a family of selection gradient $(\beta)$ functions proportional to this principal component (c). The absolute value of $\beta$ indicates the magnitude of selection, while the sign of $\beta$ indicates the direction of selection (positive or negative).

and SVL at metamorphosis and age at metamorphosis may limit evolutionary responses because size and age at metamorphosis cannot be simultaneously optimized.

Other studies on the quantitative genetics of growth have shown that genetic variation for growth trajectories is often largely confined to only a few dimensions, and 
further that most variation is for increased or decreased size across all ages. This is a general result common to investigations of artificial selection on body size (Roberts, 1961; Timon and Eisen, 1969; Rocchetta et al, 2000), discrete quantitative genetic analyses (Cheverud et al, 1983; Leamy and Cheverud, 1984), and function-valued quantitative genetic analyses (Kirkpatrick and Lofsvold, 1992; Meyer, 1998a) performed on laboratory and domesticated animals. Our results are in agreement with these studies, indicating that most genetic variation is for the position rather than the shape of the growth trajectories in A. m. columbianum as a result of consistent positive genetic covariation between sizes across ages. Another interesting aspect of our analyses is the similarity in the results obtained from principal components analysis on the genetic and phenotypic covariance functions. This similarity suggests, at least in this system, that the patterns of phenotypic variation serve as a good approximation of the patterns of genetic variation for growth trajectories.

That sizes at proximal ages should exhibit strong positive genetic covariation is perhaps not inherently surprising, but it is intriguing that this pattern of covariation is maintained across the entire measured growth trajectories in most organisms examined to date. This empirical evidence seems to suggest that in vertebrates, pronounced trade-offs for growth across ages are rare (but see Badyaev and Martin, 2000), and that the developmental processes that determine body size are often highly integrated across ontogeny. The general form of the genetic covariance functions described in this study may be relatively common across a broad range of taxa, but the evolutionary implications of genetic constraints on growth are an empirical issue based on specific selective environments. Study systems in which the fitness consequences of body size at various life stages have been relatively well characterized, such as pond-breeding amphibians, will provide an avenue for further investigations of the ways in which genetic covariance structure shapes the evolution of growth trajectories in natural populations.

\section{Acknowledgements}

We thank R Gomulkiewicz, J Kingsolver, P Verrell, R Lee, and $\mathrm{T}$ Morgan for valuable discussion, two anonymous reviewers for additional comments on the manuscript, and E Kelso and B Irwin for help with field collections. This work was supported by National Science Foundation Grant DEB 0083638 to PAC.

\section{References}

Agresti A (1990). Categorical Data Analysis. John Wiley \& Sons: New York.

Alford RA, Harris RN (1988). Effects of larval growth history on anuran metamorphosis. Am Nat 131: 91-106.

Amara R, Laffargue P, Dewarumez JM, Maryniak C, Lagardere F, Luczac C (2001). Feeding ecology and growth of O-group flatfish (sole, dab and plaice) on a nursery ground (Southern Bight of the North Sea). J Fish Biol 58: 788-803.

Arnold SJ, Pfrender ME, Jones AG (2001). The adaptive landscape as a conceptual bridge between micro- and macroevolution. Genetica 112: 9-32.

Atchley WR, Rutledge JJ (1980). Genetic components of size and shape. I. Dynamics of components of phenotypic variability and covariability during ontogeny in the laboratory rat. Evolution 34: 1161-1173.

Badyaev AV, Martin TE (2000). Individual variation in growth trajectories: phenotypic and genetic correlations in ontogeny of the house finch (Carpodacus mexicanus). J Evol Biol 13: 290-301.

Beck CW (1997). Effect of changes in resource level on age and size at metamorphosis in Hyla squirella. Oecologia 112: 187-192.

Beck CW, Congdon JD (1999). Effects of individual variation in age and size at metamorphosis on growth and survivorship of southern toad (Bufo terrestris) metamorphs. Can J Zool 77: 944-951.

Björklund M (1997). Variation in growth in the blue tit (Parsus caeruleus). I Evol Biol 10: 139-155.

Carter PA (1997). Maintenance of the Adh polymorphism in Ambystoma tigrinum nebulosum (tiger salamanders). I. Genotypic differences in time to metamorphosis in extreme oxygen environments. Heredity 78: 101-109.

Cheverud JM, Rutledge JJ, Atchley WR (1983). Quantitative genetics of development: genetic correlations among agespecific trait values and the evolution of ontogeny. Evolution 37: 895-905.

Collins JP (1979). Intrapopulation variation in the body size at metamorphosis and timing of metamorphosis in the bullfrog Rana catesbeiana. Ecology 60: 738-749.

Ebenman B (1992). Evolution in organisms that change their niches during the life-cycle. Am Nat 139: 990-1021.

Falconer DS, Mackay TFC (1996). Introduction to Quantitative Genetics. 4th edn. Longman, Essex.

Gregory TR, Wood CM (1998). Individual variation and interrelationships between swimming performance, growth rate, and feeding in juvenile rainbow trout (Oncorhynchus mykiss). Can J Fish Aquat Sci 55: 1583-1590.

Harfenist A (1995). Effects of growth-rate variation on fledging of rhinoceros auklets (Cerorhinca monocerata). Auk 112: 60-66.

Hutchings JA (1993). Adaptive life histories effected by age-specific survival and growth rate. Ecology 74: 673-684.

Kingsolver JG, Gomulkiewicz R, Carter PA (2001). Variation, selection and evolution of function-valued traits. Genetica 112: 87-104.

Kirkpatrick M, Heckman N (1989). A quantitative-genetic model for growth, shape, reaction norms, and other infinite-dimensional characters. J Math Biol 27: 429-450.

Kirkpatrick M, Lofsvold D (1992). Measuring selection and constraint in the evolution of growth. Evolution 46: 954-971.

Kirkpatrick M, Lofsvold D, Bulmer M (1990). Analysis of the inheritance, selection, and evolution of growth trajectories. Genetics 124: 979-993.

Koehl MAR (2000). Consequences of size change during ontogeny and evolution. In: Brown JH, West GB (eds). Scaling in Biology. Oxford University Press: New York, pp 67-87.

Lande R (1979). Quantitative genetic analysis of multivariate evolution, applied to brain:body size allometry. Evolution 33 402-416.

Lankford Jr TE, Billerbeck JM, Conover DO (2001). Evolution of intrinsic growth and energy acquisition rates. II. Trade-offs with vulnerability to predation in Menidia menidia. Evolution 55: 1873-1881.

Leamy L, Cheverud JM (1984). Quantitative genetics and the evolution of ontogeny.II. Genetic and environmental correlations among age-specific characters in randombred house mice. Growth 48: 339-353.

Leonard WP, Brown HA, Jones LLC, McAllister KR, Storm RM (1993). Amphibians of Washington and Oregon. Seattle Audubon Society: Seattle, Washington.

Lynch M, Walsh B (1998). Genetics and Analysis of Quantitative Traits. Sinauer, Sunderland, MA. 
Meyer K (1991). Estimating variances and covariances for multivariate animal models by restricted maximum likelihood. Genet Sel Evol 23: 67-83.

Meyer K (1998a). Estimating covariance functions for longitudinal data using a random regression model. Genet Sel Evol 30: 221-240.

Meyer K (1998b). DfReml version 3.0 \&, User Notes (http:// agbu.une.au/ kmeyen/dfreml.html)

Meyer K, Hill WG (1997). Estimation of genetic and phenotypic covariance functions for longitudinal or 'repeated' records by restricted maximum likelihood. Livest Prod Sci 47: 185-200.

Newman RA (1988). Genetic variation for larval anuran (Scaphiopus couchii) development time in an uncertain environment. Evolution 42: 763-773.

Petranka JW (1998). Salamanders of the United States and Canada. Smithsonian Institution Press, Washington, DC.

Pletcher SD, Geyer CJ (1999). The genetic analysis of agedependant traits: modeling the character process. Genetics 151: 825-835.

Ricklefs RE (1984). The optimization of growth rate in altricial birds. Ecology 65: 1602-1616.

Roberts RC (1961). The lifetime growth and reproduction of selected strains of mice. Heredity 16: 369-381.

Rocchetta G, Vanelli ML, Pancaldi C (2000). Analysis of inheritance of growth trajectories in laboratory populations of guppy-fish. Growth Dev Aging 64: 83-90.

Seibel BA, Thuesen EV, Childress JJ (1998). Flight of the vampire: Ontogenetic gait-transposition in Vampyroteuthis infernalis (Cephalopoda: Vampyromorpha). J Exp Biol 201: 2413-2424.
Semlitsch RD, Scott DE, Pechmann JHK (1988). Time and size at metamorphosis related to adult fitness in Ambystoma talpoideum. Ecology 69: 184-192.

Sever DM, Brizzi R (1998). Comparative biology of sperm storage in female salamanders. J Exp Zool 282: 460-476.

Shao J (1988). Bootstrap variance and bias estimation in linear models. Can J Stat 16: 371-382.

Shaw RG (1987). Maximum-likelihood approaches applied to quantitative genetics of natural populations. Evolution 41: 812-826.

Steppan SJ, Phillips PC, Houle D (2002). Comparative quantitative genetics: evolution of the G matrix. Trends Ecol Evol 17: 320-327.

Tejedo M, Reques R (1994). Does larval growth history determine timing of metamorphosis in anurans? A field experiment. Herpetologica 50: 113-118.

Timon VM, Eisen EJ (1969). Comparison of growth curves of mice selected and unselected for postweaning gain. Theor Appl Gen 39: 345-351.

Travis J (1980). Phenotypic variation and the outcome of interspecific competition in hylid tadpoles. Evolution 34: 40-50.

Travis J, Emerson SB, Blouin M (1987). A quantitative analysis of larval life-history traits in Hyla crucifer. Evolution 41: 145-156.

Via S, Lande R (1985). Genotype-environment interaction and the evolution of phenotypic plasticity. Evolution 39: 505-522.

Wilbur HM, Collins JP (1973). Ecological aspects of amphibian metamorphosis. Science 182: 1305-1314.

Werner EE (1986). Amphibian metamorphosis: growth rate, predation risk, and the optimal size at transformation. Am Nat 128: 319-341. 